\title{
Renal Perfusion in Scleroderma Patients Assessed by Microbubble-Based Contrast-Enhanced Ultrasound
}

\author{
Stefan Kleinert ${ }^{*}, 1$, Petra Roll ${ }^{1}$, Christian Baumgaertner ${ }^{1}$, Andrea Himsel ${ }^{2}$, Adelheid Mueller ${ }^{3}$, \\ Martin Fleck ${ }^{3}$, Martin Feuchtenberger ${ }^{1}$, Manfred Jenett ${ }^{4}$ and Hans-Peter Tony ${ }^{1}$ \\ ${ }^{1}$ University Hospital of Wuerzburg, Rheumatology/Clinical Immunology, Oberduerrbacherstr. 6, D-97080 Wuerzburg, \\ Germany \\ ${ }^{2}$ Klinikum der J.W. Goethe-Universitaet, Medizinische Klinik II/Rheumatologie, Theodor-Stern-Kai 7, 60590 Frankfurt \\ am University of Regensburg and Asklepios Clinic Bad Abbach, Germany \\ ${ }^{3}$ University of Regensburg and Asklepios Clinic Bad Abbach, Dept. of Rheumatology and Clinical Immunology, Kaiser- \\ Karl-V.-Allee 3, 93077 Bad Abbach, Germany \\ ${ }^{4}$ University Hospital of Wuerzburg, Institute of Radiology, Rheumatology/Clinical Immunology, Oberduerrbacher Str. 6, \\ D-97080 Wuerzburg, Germany
}

\begin{abstract}
Objectives: Renal damage is common in scleroderma. It can occur acutely or chronically. Renal reserve might already be impaired before it can be detected by laboratory findings. Microbubble-based contrast-enhanced ultrasound has been demonstrated to improve blood perfusion imaging in organs. Therefore, we conducted a study to assess renal perfusion in scleroderma patients utilizing this novel technique.
\end{abstract}

Materials and Methodology: Microbubble-based contrast agent was infused and destroyed by using high mechanical index by Siemens Sequoia (curved array, 4.5 MHz). Replenishment was recorded for 8 seconds. Regions of interests (ROI) were analyzed in renal parenchyma, interlobular artery and renal pyramid with quantitative contrast software (CUSQ 1.4, Siemens Acuson, Mountain View, California). Time to maximal Enhancement (TmE), maximal enhancement $(\mathrm{mE})$ and maximal enhancement relative to maximal enhancement of the interlobular artery $(\mathrm{mE} \% \mathrm{~A})$ were calculated for different ROIs.

Results: There was a linear correlation between the time to maximal enhancement in the parenchyma and the glomerular filtration rate. However, the other parameters did not reveal significant differences between scleroderma patients and healthy controls.

Conclusion: Renal perfusion of scleroderma patients including the glomerular filtration rate can be assessed using microbubble-based contrast media.

Keywords: Scleroderma, renal perfusion, contrast-enhanced ultrasound.

\section{INTRODUCTION}

Renal damage is common in connective tissue disease. In systemic sclerosis intimal edema and proliferation leads to narrowing and obliteration of small vessels. Acute renal failure (scleroderma renal crisis) or slow worsening of renal function can occur. Autopsy studies suggest that 60 to 80 per cent of patients with scleroderma have associated kidney disease $[1,2]$. Renal reserve might already be impaired before clinical renal disease is detectable [3]. Furthermore, there is no laboratory or other method to predict scleroderma renal crisis. Improved imaging of renal perfusion using contrastenhanced ultrasound has been described previously [4]. We therefore conducted a pilot study to assess renal perfusion

*Address correspondence to this author at the University Hospital of Wuerzburg, Rheumatology/Clinical Immunology, Oberduerrbacherstr. 6, D97080 Wuerzburg, Germany; Tel: +49 931201 40100; Fax: +49 931201 640100; E-mail: Kleinert_S@klinik.uni-wuerzburg.de by contrast-enhanced ultrasound in patients with scleroderma compared to healthy controls.

\section{MATERIALS AND METHODOLOGY}

After approval by the local ethics committee, ultrasonographic examinations were conducted in scleroderma patients compared to healthy volunteers as age and sex matched control. Siemens Sequoia (curved array, 4.5 $\mathrm{MHz}$ ) was used for analysis, and kidneys were documented in B-Mode. The resistance index of the interlobular arteries was measured by color Doppler ultrasound. Measurement of the intrarenal resistance index has been widely established in the evaluation of renovascular changes and is a wide-spread method to get indirect information about the vessel resistance and the perfusion distal of the measuring point. 10 millilitres ( $\mathrm{ml}$ ) of Sonovue ${ }^{\circledR}$ contrast agent (Altana Pharma) were infused at $90 \mathrm{ml}$ per hour. Sonovue is a suspension of phospholipid stabilized sulphur hexafluoride (SF6) microbubbles. $90 \%$ of microbubbles are smaller than $8.0 \mu \mathrm{m}$, 
(mean diameter $2.5 \mu \mathrm{m}$ ) and have a strong echogenicity over the range of frequencies used in medical ultrasound examinations. Ultrasound was performed with the Sequoia CPS programme with a mechanical index of 0.21 . After saturation of Sonovue in the kidneys a high mechanical index was used to destroy the contrast medium locally. Replenishment was recorded for 8 seconds. Regions of interests (ROI, Figs. 1-3) were analyzed in renal parenchyma, interlobular artery and renal pyramid with quantitative contrast software (CUSQ 1.4, Siemens Acuson, Mountain View, California) using a typical exponential function as estimation [5]. Slope, time to maximal Enhancement (TmE), maximal enhancement (mE) and maximal enhancement relative to maximal enhancement of the interlobular artery $(\mathrm{mE} \% \mathrm{~A})$ were calculated for different ROIs. Graphical and statistical analyses were performed by Pearson Correlation (two-sided confidence interval 95\%) and Mann-Whitney test to compare between control and Scleroderma group using the software GraphPad Prism 3.02. Graphs are displayed with single patients' results and median, minimum, maximum, and $25^{\text {th }}$ and $75^{\text {th }}$ percentile on the right side. Patients were characterized by autoantibody profile and concomitant medication. Modified Rodnan skin score was assessed. This score increases from 0 to 51 in severity and there is an association between severe skin involvement and burden of organ-based complications.

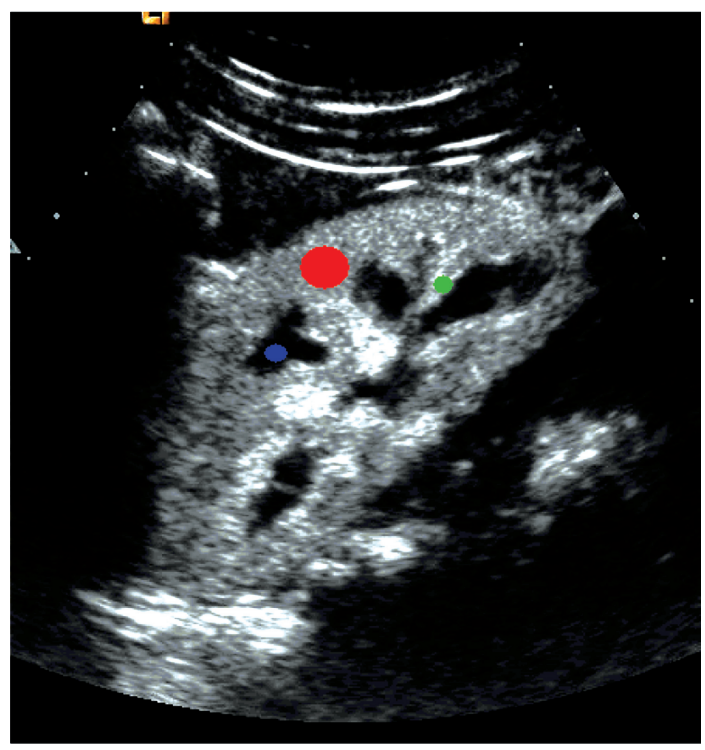

Fig. (1). Regions of interest: Red - parenchyma, green interlobular artery, blue - renal pyramid.

\section{RESULTS}

\section{Patient Characteristics}

Median (mean) age of healthy controls $(n=12)$ was 49.5 (50.6) years, Scleroderma patients $(n=14)$ had a median age of 43.5 (42.4) years ( $p=0,1498$; n.s.). Nine patients suffered from the diffuse form of systemic sclerosis, whereas 5 patients presented with a limited form. Six patients were tested positive for antibodies against $\operatorname{Scl70}(70 \mathrm{kD}$ extractable immunoreactive fragment of anti-topoisomerase I), 2 patients against centromere protein B (CENP-B), and 1 against ribonucleic acid (RNA) polymerase. Mean (median) disease duration was 6.1 (5.5) years. Eight patients had at least one digital ulcus at the time of examination. Seven patients had pulmonary manifestations, all patients had esophageal involvement. Three patients revealed a reduction in glomerular filtration rate below $80 \mathrm{ml} / \mathrm{min}$. Five patients were treated with an angiotensin-converting-enzyme (ACE) inhibitor. In the group of healthy controls, no renal dysfunction became evident by labaratory testing and treatment with an ACE inhibitor was not reported. Inspite of this, there was no significant difference in glomerular filtration rate between both groups (estimated by using Modification of Diet in Renal Disease (MDRD) formula [6], validated only below $90 \mathrm{ml} / \mathrm{min}, \mathrm{p}=0,52$ ).

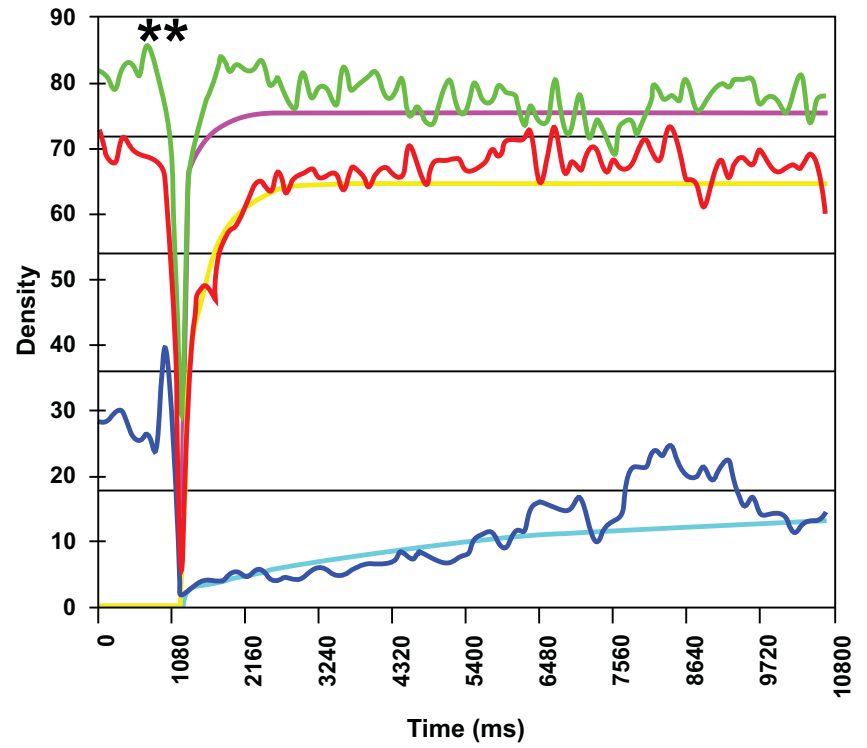

Fig. (2). Example of characterization of renal perfusion in different regions of interest. Destruction of microbubbles (**). Maximum enhancement (plateau) in renal parenchyma (red line, line of best fit yellow) is close to maximum enhancement in the interlobular artery (green line, line of best fit pink). Blue line (line of best fit blue): renal pyramids.

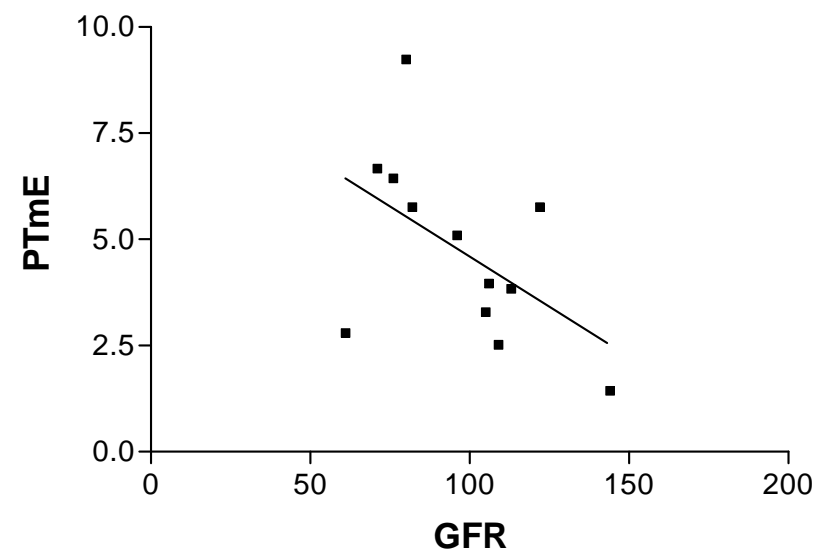

Fig. (3). Pearson's Correlation between PTmE (time to maximal enhancement in renal parenchyma) and glomerular filtration rate (GFR) assessed by MDRD. 
Six patients were treated with corticosteroids of more than $5 \mathrm{mg}$ of prednisone equivalent per day at the time of examination. All except one in the patient group were female. The mean (median, SD) modified rodnan skin score was determined at $10.8(7.5,9.0)$.

\section{Color Doppler Ultrasound}

Resistance index was analyzed in the interlobular arteries. There were no significant differences between both groups (data not shown).

\section{Renal Perfusion Assessed by Microbubble-Based Contrast-Enhanced Ultrasound}

Renal perfusion using Sonovue was recorded and afterwards quantified as described above.

Renal perfusion assessed by microbubble-based contrastenhanced ultrasound revealed a linear correlation between time to maximal enhancement in renal parenchyma (PTmE) and glomerular filtration rate (GFR) assessed by MDRD that was close to statistical significance. The correlation coefficient using Pearson's linear correlation was determined at -0.52 (CI $0.95 \%,-0.8419$ to 0.07911 ) with $\mathrm{p}=0.08$ (Fig. 4).

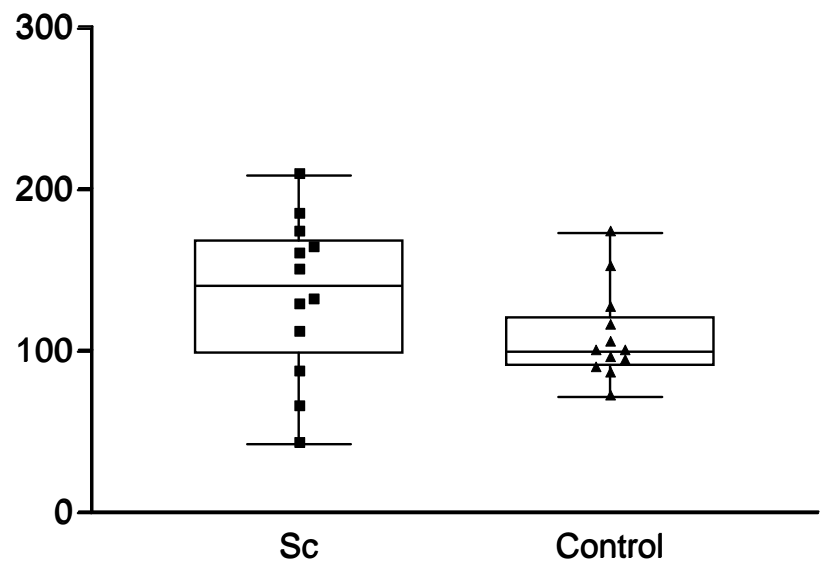

Fig. (4). Maximal enhancement in renal parenchyma relative to maximal enhancement interlobular arteries $(\mathrm{mE} \% \mathrm{~A})$.

However, there were no statistically significant differences regarding time to maximal enhancement in renal parenchyma (PTmE, data not shown) or artery (ATmE, data not shown), maximal enhancement in renal parenchyma (PmE, data not shown), maximal enhancement in parenchyma relative to maximal enhancement of the interlobular artery $(\mathrm{mE} \% \mathrm{~A}$, Fig. 4), and slope in renal parenchyma (first ascending part of the curve resembling the speed of blood moving into the selected region (Fig. 5).

\section{DISCUSSION}

Estimation of renal perfusion by microbubble-based contrast media was established in a phantom [5]. Contrastenhancend ultrasound has been demonstrated to detect perfusion differences in transplanted kidneys that were about to be rejected compared to non-rejected kidneys [7], and to determine other causes of delayed graft function [8]. Applying this novel technique to scleroderma patients to assess renal function, we were able to reveal a correlation between GFR and the time until maximal enhancement of

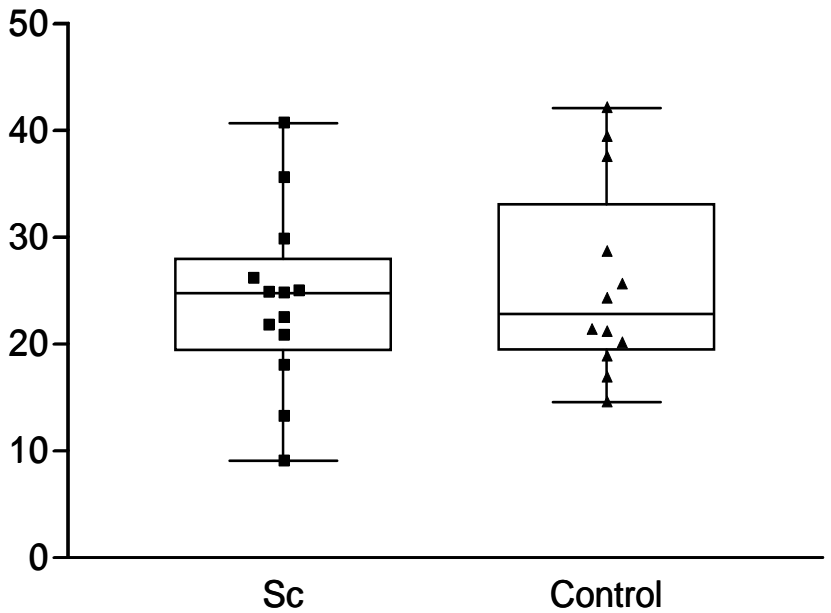

Fig. (5). Quantification of renal perfusion by slope in renal parenchyma (slope).

microbubbles in the renal parenchyma (PTmE). However, some limitations need to be considered when evaluating this finding. The correlation was only close to statistical significance and a larger cohort of scleroderma patients is required to further analyze the correlation and find out whether this novel method might provide substantial information beyond the glomerular filtration rate. There was no dose finding study ahead and the level of microbubble saturation might have influence on test results. There is a limitation of this calculation as the GFR estimated by MDRD was mainly established in a patient cohort presenting with a GFR below $90 \mathrm{ml} / \mathrm{min}$. In a very small previously published scleroderma group, there were significant differences observed in maximal enhancement in parenchyma relative to maximal enhancement in interlobular arteries (Pmax\%A) compared to normal controls [9]. This parameter suggests a higher independence of renal perfusion since this parameter is calculated as an intra-individual relation of perfusion that might be independent from the filling of the extrarenal vessels. However, in our study, we were not able to confirm these differences in Pmax\%A (Fig. 4). We could neither demonstrate differences in time until maximal enhancement in the parenchyma (PTmE) nor in the interlobular arteries (ATmE) compared to healthy controls. Neither maximal enhancement in the renal parenchyma (PmE) nor the height of the slope in parenchyma (slope, Fig. 5) revealed significant differences compared to healthy controls. Therefore, no additional parameter could be identified to assess subclinical renal changes. Nevertheless, our pilot study suggests the feasibility to assess renal perfusion including the glomerular filtration rate of scleroderma patients by microbubble-based contrast media.

\section{ACKNOWLEDGEMENTS}

We are grateful for financial support of the study by "Deutsche Stiftung Sklerodermie" (German Scleroderma Foundation).

This publication was funded by the German Research Foundation (DFG) and the University of Wuerzburg in the funding program Open Access Publishing. An unrestricted research grant by Bracco Altana Pharma that was used to cover fees for patient insurance. 


\section{COMPETING INTEREST}

M. Jenett received lecture fees from Siemens and Altana Pharma and an unrestricted research grant by Bracco Altana Pharma. All other authors: None.

\section{REFERENCES}

[1] Medsger TA Jr, Masi AT, Rodnan GP, Benedek TG, Robinson H. Survival with systemic sclerosis (scleroderma). A life-table analysis of clinical and demographic factors in 309 patients. Ann Intern Med 1971; 75(3): 369-76.

[2] Medsger TA Jr, Masi AT. Survival with scleroderma. II. A lifetable analysis of clinical and demographic factors in 358 male U.S. veteran patients. J Chronic Dis 1973; 26(10): 647-60.

[3] Livi R, Teghini L, Pignone A, Generini S, Matucci-Cerinic M, Cagnoni M. Renal functional reserve is impaired in patients with systemic sclerosis without clinical signs of kidney involvement. Ann Rheum Dis 2002; 61(8): 682-6.
[4] Quaia E. Quantitative Analysis of Renal Perfusion at ContrastEnhanced US. In: Quaia E, Ed. Cotrast Media in Ultrasonography. Berlin, Heidelberg, New York: Springer 2005.

[5] Lucidarme O, Franchi-Abella S, Correas JM, Bridal SL, Kurtisovski E, Berger G. Blood flow quantification with contrast-enhanced US: "entrance in the section" phenomenon--phantom and rabbit study. Radiology 2003; 228(2): 473-9.

[6] Levey AS, Bosch JP, Lewis JB, Greene T, Rogers N, Roth D. A more accurate method to estimate glomerular filtration rate from serum creatinine: a new prediction equation. Modification of Diet in Renal Disease Study Group. Ann Intern Med 1999; 130(6): 461-70.

[7] Fischer T, Muhler M, Kroncke TJ, et al. Early postoperative ultrasound of kidney transplants: evaluation of contrast medium dynamics using time-intensity curves. Rofo 2004;176(4): 472-7.

[8] Grzelak P, Szymczyk K, Strzelczyk J, et al. Perfusion of kidney graft pyramids and cortex in contrast-enhanced ultrasonography in the determination of the cause of delayed graft function. Ann Transplant 2011;23(16): 48-53

[9] Kleinert S, Baumgaertner C, Feuchtenberger M, et al. Changes in renal perfusion in patients with systemic sclerosis. Arthritis Rheum 2005; 52(9): S591.

This is an open access article licensed under the terms of the Creative Commons Attribution Non-Commercial License (http://creativecommons.org/licenses/by-nc/ $3.0 /$ ) which permits unrestricted, non-commercial use, distribution and reproduction in any medium, provided the work is properly cited. 